\title{
Prediction of surface roughness in low speed turning of AISI316 austenitic stainless steel
}

\author{
Acayaba, Gabriel Medrado Assis \\ Department of Production Engineering, University of São Paulo \\ Muñoz de Escalona, Patricia \\ Department of Mechanical and Aerospace Engineering, University of Strathclyde
}

January 20, 2015

\begin{abstract}
Surface roughness is an important quality in manufacturing, as it affects the product's tribological, frictional and assembly characteristics. Turning stainless steel at low cutting speeds may result in a rougher surface due to built up edge formation, where as speed increases the surface roughness improves, due to the low contact time between the chip and the tool to allow bonding to occur.However, this increase in cutting speed produces higher tool wear rates, which increases the machining costs.

Previous studies have indicated that savings in cost and manufacturing time are obtained when predicting the surface roughness, prior to the machining process. In this paper, experimental data are used to develop prediction models using Multiple Linear Regression and Artificial Neural Network methodologies. Results show that the neural network outperforms the linear model by a fair
\end{abstract}


margin(1400\%). Moreover, the developed Artificial Neural Network model has been integrated with an optimisation algorithm, known as Simulated Annealing (SA), this is done in order to obtain a set of cutting parameters that result in low surface roughness. A low value of surface roughness and the set of parameters resulting on it, are successfully yielded by the SA algorithm.

Keywords: Stainless Steel, Turning, Surface Roughness, Multiple Linear Regression, Artificial Neural Networks, Simulated Annealing.

\section{Introduction}

In order to achieve the nominal values of surface roughness specified by engineering design, the right combination of machining parameters must be chosen by manufacturing. In any cutting process, the geometry, the tolerances and the surface roughness of the machined piece are very important, as they represent the quality of the process.

The surface roughness in a turning process is affected by many factors such as: the geometry of the cutting tool, the depth of cut, the cutting speed, the feed rate, the workpiece's microstructure and the rigidity of the lathe (Gokkaya, H. et al. 2007).The surface finish also affects considerably the performance of produced machines, since many aspects of equipment's performance such as desired efficiency, mechanical life, and the resistance against environmental factors are influenced by it. Economic losses arise when the working parameters are not selected properly. For this reason, much effort has been directed in understanding the effects of cutting conditions on the quality of the machined surface and to the creation of adequate models which can be used to find optimal or near-optimal cutting parameters for objectives such as obtaining a desired value of surface roughness, surface integrity, reduction of machining time, tool wear, and many others. However, not much focus has been given to the prediction of surface roughness at low speed turning. Low speed turning may become the best choice in cases where advantages related to a decrease in tool wear and consequently on the 
decrease of machining costs can be mentioned. This research studies the effects of cutting parameters on the surface roughness when turning AISI316 austenitic stainless steel, a widely used material in Precision Engineering applications, such as petrochemical and brewing industry, piping and connections, medical and surgical instruments, and hydraulic systems.

To help manufacturing engineers in the decision making process for reducing time and cost of cutting process, researchers propose models that try to simulate the conditions during the machining process, establishing the cause and effect relationships between various factors and desired product characteristics. Furthermore, the technological advances in the field, for instance the ever-growing use of computer controlled machine tools, have brought up new issues to deal with, emphasizing the need for more precise predictive models, hence many authors have worked on prediction models for surface roughness. Among these researchers we can mention Benardos and Vosniakos, 2013. These researchers carried out an extensive literature review on the subject and four major categories were created to classify the selected papers. These are: (i) approaches that are based on machining theory to develop analytical models and/or computer algorithms to represent the machined surface; (ii) approaches that examine the effects of various factors through the execution of experiments and the analysis of the results; (iii) approaches that use designed experiments; and (iv) artificial intelligence (AI) approaches. The present research uses approach (ii) when analysing experimental results and developing the regression model, since it carries out statistical analysis on experimental results, and approach (iv) when Artificial Neural Networks are used to model the surface roughness behaviour and Simulated Annealing is used to select the cutting parameters. In approaches (ii) and (iv), no analytical models based on machining theory are used, since they use empirical performance equations established from extensive testing. According to Benardos and Vosniakos, 2013, models based on theory are generally not accurate so their improvement with the introduction of additional parameters is examined by researchers. This is due to the fact that the phenomena that lead to the formation of surface roughness are very 
complex and interacting in nature so a comprehensive solution has not yet been found. The objective of this research is to develop a methodology for predicting the surface roughness and selecting parameters based on effective tools, hence the use of empirical data.

Other researchers have focused their effort on the analysis of the machinability of austenitic stainless steel. This material is considered difficult to machine due to its low thermal conductivity and high mechanical and microstructural sensitivity to strain and stress rate (M'Saoubi et al.,2008). Different works have been developed to improve the machinability of austenitic stainless steel, and among these researchers we can mention Lin (2008), where the surface roughness variations for different grades of austenitic stainless steel under different cutting conditions in high speed fine turning was investigated. The surface roughness and the tool wear were predicted by Regression analysis and ANOVA.

In 2009, Anthony Xavior and Adithan determined the influence of different cutting fluids on the wear and the surface roughness when turning AISI 304 austenitic stainless steel. Ibrahim Ciftci (2006) conducted experiments to machine AISI 304 and AISI 316 austenitic stainless steels using CVD multi-layer coated cemented carbide tools. The study concluded that cutting speed significantly affected the machined surface roughness. Cebeli et al., 2006 conducted an investigation to determine surface roughness, tool wear and tool-chip interface temperature during turning of AISI 304.

Models for tool life, surface roughness and cutting forces were developed for turning of AISI302 by Al-Ahmari (2007), using multiple regression analysis techniques, response surface methodology and computational neural networks. Ulas Caydas and Sami Ekici (2010) used support vector machines (SVM) tools, namely least square-SVM, spider SVM and artificial neural networks (ANN) models to assess the surface roughness of AISI 304 austenitic stainless steel. Akasawa T. et al (2003) conducted experiments to determine the effect of variation of 
concentrations of $\mathrm{S}, \mathrm{Ca} \mathrm{C}$ and $\mathrm{Bi}$ on the machinability of various grades of 300 series of austenitic stainless steel.

Models based on Linear Regression have also been applied with success by researchers in more recent years. The relationship between tool life, surface roughness and vibration was examined by O.B. Abouelatta, J. Madl, in 2001.In these case the variables that were considered included the cutting speed, feed rate, depth of cut, tool nose radius, tool overhang, approach angle, workpiece length and workpiece diameter. The experimental data was analysed to produce regression analysis models. R. Baptista, J.F. Antune Simoes, 2000, applied Design of Experiments technique together with multiple linear regression to establish a mathematical model of 3 and 5 axes milling process. A comprehensive literature review on the applications of ANNs for turning process performance prediction can be foundat Chandrasekaran et al, 2010.According to this paper, Simulated Annealing (SA) imitates the cooling process of metal during annealing to achieve the minimization of a given function values. The algorithm starts with an initial point, $\mathrm{X}_{0}$, which is a set of input data for the fitness function, and a large number corresponding to a high temperature " $\mathrm{T}$ ". A second point $\mathrm{x}_{1}$ is generated close to the first point using a Gaussian distribution with first point as its mean. The difference in the fitness function values at these points is considered analogous to the difference in energy level $(\Delta \mathrm{E})$. In a minimization process, if the second point has lower function value, it replaces the first point; otherwise, it replaces the first point with a probability $\exp (-\Delta \mathrm{E} / \mathrm{T})(\mathrm{Deb}, 1995)$.The algorithm is stopped when a sufficiently small temperature is obtained or no significant improvement in the fitness function value is observed. Chen and Tasi,1996, followed by many researchers applied SA to solve the optimization problem for minimum unit production cost of multi-pass turning process. Baykasoglu and Dereli, 2002, have used SA to optimize cutting conditions in their heuristic model. However, they did not take surface finish into consideration. 
Once analysing the literature review, this research will be focused on comparing the performance of the Multiple Linear Regression and the Artificial Neural Network methodologies for the prediction of the surface roughness and the use of the best fitting method as the objective function in an SA algorithm. This will provide a novel contribution to the manufacturing field by giving the opportunity to find a good procedure to model and obtain the desired surface roughness when turning at low cutting speed.

\section{Methodology}

A cylindrical sample of austenitic stainless steel with $200 \mathrm{~mm}$ length and $30 \mathrm{~mm}$ in diameter was selected for this investigation. The bar was pre-machined in 10 subsections corresponding to different combination of cutting parameters, as observed in Figure 1.

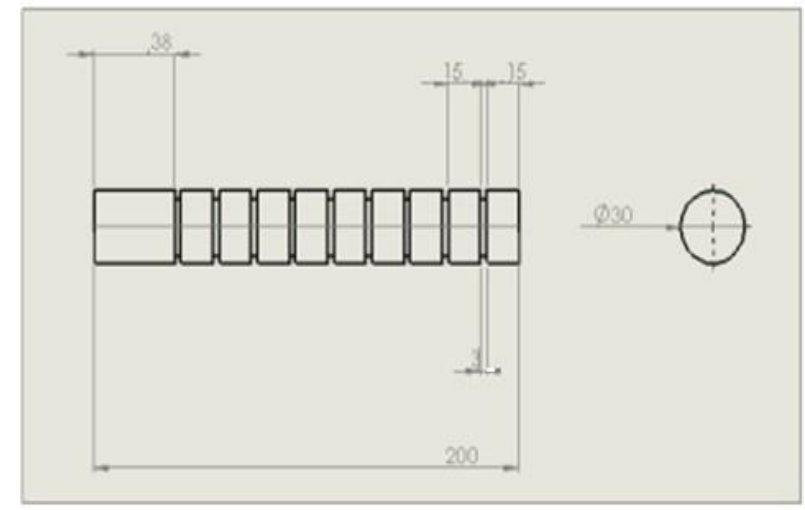

Figure 1: Schematic drawing of workpiece used in the experiments .

The austenitic stainless steel has a chemical composition of $17.1 \% \mathrm{Cr}, 68.7 \% \mathrm{Fe}$, 9.9\% Ni, 2.1\% Mo, 1.8\% Mn and $0.47 \% \mathrm{Si}$, which was obtained through the use of a 
Hitachi S-3700N Scanning Electron Microscope coupled with an Oxford Inca 350 EDX analyser.

According to ASM Metals Handbook, the mechanical properties of AISI316 are as follows:

Table 1: Mechanical Properties of AISI316

\begin{tabular}{|c|c|}
\hline Vickers Hardness & $258 \mathrm{HV}$ \\
\hline Tensile Strength, Yield & $290 \mathrm{MPa}$ \\
\hline Tensile Strength, Ult. & $580 \mathrm{MPa}$ \\
\hline Elongation at break & $50 \%$ \\
\hline Modulus of Elasticity & $193 \mathrm{GPa}$ \\
\hline
\end{tabular}

HERTEL inserts with TiN/TiAlN carbide coating with two different tool nose radius; $0.4 \mathrm{~mm}$ and $0.8 \mathrm{~mm}$ were selected for the study. Figure 2 shows the geometry and dimension of the inserts.

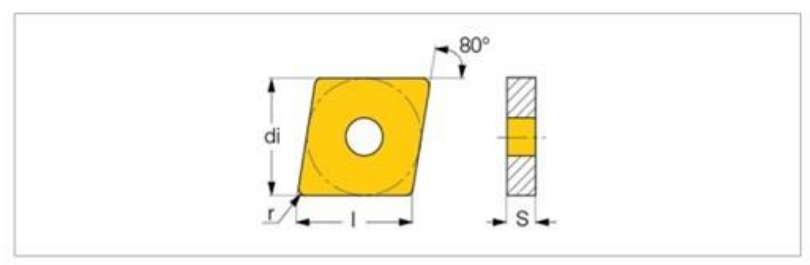

Figure 2: Schematic drawings of turning insert geometry

Table 2 shows the cutting parameters selected for the study. These parameters were recommended by the tool supplier based on the tool-workpiece combination.A XYZ Proturn SLX 1630CNC Lathe with a maximum spindle of $2500 \mathrm{rpm}$, was selected for the turning experiments. The cutting process was conducted under a dry cutting environment and a new tool was used to cut a length of $200 \mathrm{~mm}$ under different combination of cutting parameters ( 10 different speeds and constant depth of cut, feed rate and tool nose radius). 
Table 4: Testing parameters

\begin{tabular}{lllllllllll}
\hline Cutting Speed(m/min) & 14 & 15 & 16 & 18 & 20 & 22 & 25 & 30 & 40 & 50 \\
\hline Feed rate $(\mathrm{mm} / \mathrm{rev})$ & 0.15 & 0.20 & 0.25 & & & & & & \\
\hline Depth of cut $(\mathrm{mm})$ & 0.5 & 1.0 & & & & & & & & \\
\hline Tool nose radius $(\mathrm{mm})$ & 0.4 & 0.8 & & & & & & & \\
\hline
\end{tabular}

Once the material was machined it was placed in a bench for surface roughness measurements. The surface roughness was measured across the tool feed direction using a Mitutoyo Surftest- SV2000 profilometer, with the cut-off length at $15 \mathrm{~mm}$ providing a three sample length in compliance with ASME B46.1 and to avoid noisy data. An example of the measured surface roughness values can be found in the appendices (Section 8).

\section{Results and discussion}

Once the experimental data was gathered, in order to minimise the effect of noisy data, the values that exceeded two standard deviations from the population's average were not considered to take part of the analysis. In a normal probability distribution, 95\% of population is contained within two standard deviations above and below the average. The remaining 5\% were discarded from further analysis to avoid noisy data propagation. This is based on the Tchebysheff 's theorem (Kvanli et al, 2006).

The two models for surface roughness prediction were developed from the experimental data. The first model is based on a multiple linear regression. The second model is based on an Artificial Neural Network. A comparison of both methods can be found in the following sections. 


\subsection{Multiple Linear Regression}

The multiple linear regression (MLR) model was developed with the aid of the statistical software Minitab 16. Different adjustments were used (linear, exponential, logarithmic) and the adjustment that presented the best coefficient of correlation was chosen. The proposed regression equation with potential adjustment is given by:

$$
\begin{aligned}
& \mathrm{R}_{\mathrm{a}}=10^{1.31} \mathrm{~V}^{-0.424} \mathrm{f}^{0.734} \mathrm{~d}^{0.187} \mathrm{r}^{-0.499}(1) \\
& \mathrm{R}^{2}=0.466 \\
& \mathrm{R}_{\text {adj }}^{2}=0.460 \\
& \sigma=1.33
\end{aligned}
$$


The squared multiple correlation coefficient $\left(\mathrm{R}^{2}\right)$ is the proportion of the variation in the response variable that is explained by the response variables.(Glantz and Slinker, 2000). The adjusted coefficient $\mathrm{R}_{\text {adj }}^{2}$ of equation 1 is 0.46 when applied to the experimental data of this research, (i.e. it explains $46 \%$ of variability within this dataset).

The mean squared error (MSE) is an estimator commonly used to measure the squares of the errors, (i.e. the difference between the predicted and actual values of a statistical variable(Lehmann et al, 1998). If $\hat{y}$ is a vector of $n$ predictions, and $y$ is the vector of the true values, then the estimated mean squared error of the predictor isas shown in equation 2.

$$
M S E=\frac{1}{n}\left(\widehat{y}_{l}-y_{i}\right)
$$

$M S E$ was used to evaluate the regression model, resulting in the value of $M S E=0.72$, which means the regression model is a fairly good approximation to the real behaviour of the system (the closer MSE is to 0 , the better the model). The calculations of mean squared errors can be found at section 8 .

According to the developed model shown in equation 1, the lowest predicted Ra value is $0.339 \mu \mathrm{m}$ at $\mathrm{V}=50 \mathrm{~m} / \mathrm{min}, \mathrm{f}=0.15 \mathrm{~mm} / \mathrm{rev}, \mathrm{d}=1.0 \mathrm{~mm}$ and $\mathrm{r}=0.8 \mathrm{~mm}$. This is not a good estimate, since the measured $\mathrm{Ra}$ value for this set of parameters is $2.2 \mu \mathrm{m}$, which is far from the estimated value and is not the lowest measured value found in the experimental dataset. Therefore, it can be concluded that the regression model is good for prediction in general, but it is not suitable for finding optimal parameters. 


\subsection{Artificial Neural Network}

Figure 3 show a diagram depicting the selected network, where $\mathrm{W}$ stands for weights and $\mathrm{b}$ stands for biases.

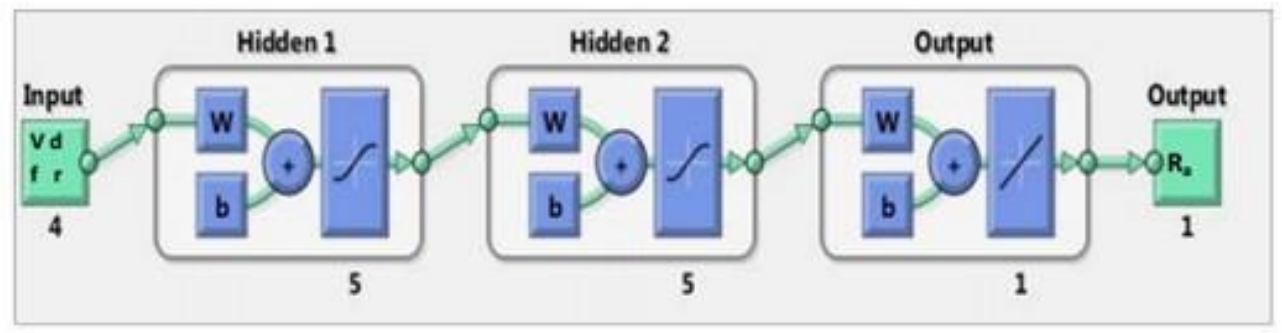

Figure 3: Schematic of the MLP created to predict the surface roughness from the experimental results.

The experimental results data set was divided randomly in three groups from a total of 363 values: 273 values for training, 54 values for testing and 36 values for validation process. The training data are presented to the network during training, and the network is adjusted according to its error. It must be highlighted that the testing data have no effect on training so it provides an independent measure of the network performance during and after the training process. The validation data are used to measure the network generalization, and to halt training when generalization stops improving.(Muñoz-Escalona et al, 2010)

In this case the Levenberg-Marquardt back propagation algorithm (LMA) was used for the training purpose. The LMA interpolates between the Gauss-Newton algorithm (GNA) and the method of gradient descent. The LMA was chosen because it is more robust than the GNA, which means that in many cases it finds a solution even if it starts very far off the final minimum.

The stopping criterion for training was obtained when generalization stopped improving. This is indicated by an increase in the mean square error of the values selected for the validation process.

Figure 4 shows the correlation plot between experimental and predicted data. As observed when analyzing Figure 4, there is a good fit between the network's outputs and the target (measured) values. 


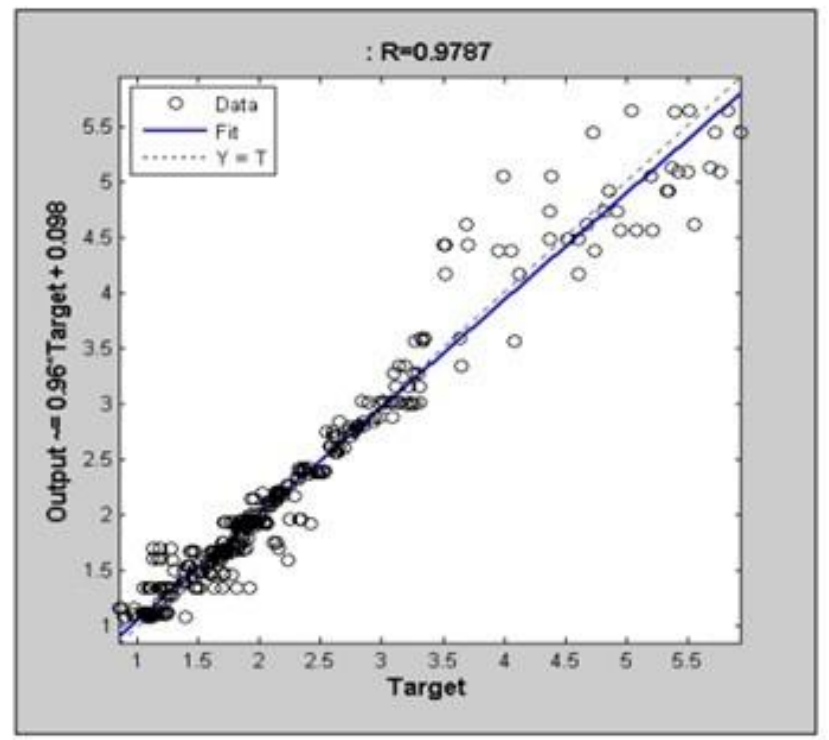

Figure 4: Regression plot for MLP outputs vs. targets.

The results in terms of $M S E$ for each phase of the network were as follows: a training performance of 0.0489 , testing performance of 0.0636 and validation performance of 0.0472 , yielding a general performance of 0.0509 . These values are considered as very good as they outperform the Multiple Linear Regression results by more than 14 times (1400\%). The networks outputs as well as the MSE values for each point can be found in the appendices (section 8).

When comparing the performance of the two prediction models for surface roughness employed by their general MSE, it can be concluded that ANN has a considerably better performance when compared to the MLR when predicting the surface roughness for AISI316 turning, since the MSE obtained for the ANN was 0.05 which is nearly fourteen times smaller (1400\%) than the MLR which obtained a 0.72 MSE. These results are in agreement with previous research such as Chryssolouris and Guillot, 1990; Jiao et al, 2005 and Al-Ahmari, 2007, who found that ANNs are better than MLR for predicting surface roughness in turning processes. However, Feng and Wang, 2003, found that the ANN and the MLR are equally effective in predicting the surface roughness. Since from our results the ANN model displayed the best performance, this model will be used as the fitness function for a Simulated Annealing (SA) algorithm. 


\section{Cutting parameters selection}

In order to find parameters that return low values of surface roughness (below $1.0 \mu \mathrm{m}$ ), the proposed ANN has been used as the fitness function in a Simulated Annealing (SA) algorithm set for the minimisation of the surface roughness values. The problem was set up in MATLAB R2011a using the Optimisation Toolbox. The objective function's lower limit was set to 0 , since Ra values are always positive. Other parameters were set as the default SA parameters on MATLAB's Optimisation Toolbox.

The maximum number of iterations was set as infinite, maximum function evaluations was 12000 and no time limit was specified. The function tolerance, the lowest significant improvement before the algorithm stops, was set to 1e-6. The number of stall iterations was set as 2000 . In order to apply the SA method to a specific problem, the following parameters must be also specified: the annealing schedule temperature, the re-annealing interval, the temperature update function and the initial temperature (Kirkpatrick, S. et al , 1983). These parameters are given this nomenclature due to their analogy with the metal cooling process. The fast annealing function was used with a re-annealing interval of 100 , exponential temperature update and initial temperature of 100. The SA algorithm must have an initial point from which the iterations are calculated. The closer this initial point is to the actual solution of the SA, the faster the convergence of the algorithm and the shortest the calculation time. Therefore, a good initial "guess" must be provided. In this work, the initial point was set as the best point found by the regression model. The upper and lower bounds are based on the upper and lower values of each of the four parameters used in this work: cutting speed, feed rate, depth of cut and tool nose radius. These points can be seen in Table 5:

Table 5: Inputs for SA algorithm

\begin{tabular}{ccccc}
\hline Point & $\mathrm{V}(\mathrm{m} / \mathrm{min})$ & $\mathrm{f}(\mathrm{mm} / \mathrm{rev})$ & $\mathrm{d}(\mathrm{mm})$ & $\mathrm{r}(\mathrm{mm})$ \\
\hline Initial point & 50 & 0.15 & 0.5 & 0.8 \\
\hline Lower bound & 14 & 0.15 & 0.5 & 0.4 \\
\hline Upper bound & 50 & 0.25 & 1.0 & 0.8 \\
\hline
\end{tabular}


The fitness function value and the correspondent set of parameters generated by the algorithm are in Table 6.

Table 6: SA algorithm's outputs.

\begin{tabular}{ccrrr}
\hline $\mathrm{Ra}(\mu \mathrm{m})$ & $\mathrm{V}(\mathrm{m} / \mathrm{min})$ & $\mathrm{f}(\mathrm{mm} / \mathrm{rev})$ & $\mathrm{d}(\mathrm{mm})$ & $\mathrm{r}(\mathrm{mm})$ \\
\hline 0.65 & 49.3 & 0.156 & 0.52 & 0.57 \\
\hline
\end{tabular}

This result can be considered plausible as cutting speed is near the higher value of the interval, in concordance with the results of Lin, 2008; Ozel, 2003 and Kilickap, 2005. The combination of depth of cut and tool nose radius values obtained are in agree with Halevi, 2003 who states that as a general rule of thumb, the depth of cut should be greater than or equal to $2 / 3$ of the nose radius, in order to minimize the effect of axial forces that appear as the depth of cut increases in relation to the tool nose radius. These axial forces have a negative effect on the cutting action e.g. with more tendency to vibration and rougher surface finishing. Finally, the feed rate value is near the lower specified limit, concurring with the results of Lin, 2008; Ozel, 2003 and Kilickap, 2005, where surface roughness average values rise with higher feed rates.

\section{Conclusion}

First, it is important to note that the solutions found by the algorithm cannot be called optimal, since this method is a metaheuristic, designed to find good, near optimal solutions, but not to guarantee an optimal fitness function value.

- A Multiple Linear Regression model for predicting Ra was developed. It yielded results with 0.72 precision in terms of Mean Squared Error when compared to the experimental data. This model can be useful for pre- diction where high precision is not needed as the formula is simple and a straight forward result can be obtained.

- An Artificial Neural Network model was created to predict Ra from a given set of parameters. It was trained, tested and validated using experimental data. After many trials, a network with an accuracy of $98 \%$ was found with two hidden layers with five neurons each, which is an excellent 
result considering that there is variation of $15 \%$ on roughness under the same cutting conditions. The network's outputs are a good fit for the experimental results, and when compared in terms of mean squared error it outperforms the multiple linear regression model by a considerable margin.

- The proposed ANN was used as the fitness function in Simulated Annealing. The algorithm produced good and feasible results.

- The cutting conditions obtained by the SA algorithm for low values of surface roughness are $\mathrm{V}=$ $49.3 \mathrm{~m} / \mathrm{min}, \mathrm{f}=0.156 \mathrm{~mm} / \mathrm{rev}, \mathrm{d}=0.52 \mathrm{~mm}$ and $\mathrm{r}=0.57 \mathrm{~mm}$

\subsection{Future work}

Some research opportunities found are the possible inclusion of other factors such as vibration and cutting forces as explanatory variables and others such as tool wear and residual stresses as variables to be analysed and predicted. Response Surface Methodology (RSM) could be used to predict Ra and its performance compared to ANN and MLR. Other ANN types could be used for prediction, such as RBF and fuzzy-logic integrated networks. The genetic algorithm could be used to optimise the weights and biases of the proposed network. Other optimisation algorithms such as Particle Swarm Optimisation and Ant Colony Optimisation can be used to optimise the AN fitness function and their results compared. Finally, measurements in energy consumption, and a comparison between high and low values of cutting speeds can be carried out for the pursuit of similar values of surface roughness.

\section{Acknowledgements}

The authors wish to thank the Department of Mechanical and Aerospace Engineering of the University of Strathclyde for making available all laboratories and equipment used in this research, as well as all crew involved who dedicated their expertise and abilities to this work. We would also like to thank the funding of this project made possible by $\mathrm{CNPq}$, a public research funding organization from the government of Brazil. 


\section{Appendix}

Table 7: Experimental surface roughness values and results from Multiple Linear Regression(MLR) and Multi-Layer Perceptron(MLP) models.

\begin{tabular}{lllllllll}
\hline $\mathrm{V}(\mathrm{m} / \mathrm{min})$ & $\mathrm{f}(\mathrm{mm} / \mathrm{rev})$ & $\mathrm{d}(\mathrm{mm})$ & $\mathrm{r}(\mathrm{mm})$ & $\mathrm{R}_{\mathrm{a}}(\mu \mathrm{m})$ & $\mathrm{R}_{\mathrm{a}}(\mathrm{MLP})$ & $\mathrm{R}_{\mathrm{a}}(\mathrm{MLR})$ & Sq-err(MLP) & Sq-err(MLR \\
\hline 14 & 0.15 & 0.5 & 0.4 & 1.267 & 1.331 & 2.640 & 0.004 & 1.886 \\
15 & 0.15 & 0.5 & 0.4 & 1.187 & 1.186 & 2.604 & 0.000 & 2.006 \\
16 & 0.15 & 0.5 & 0.4 & 1.113 & 1.135 & 2.567 & 0.000 & 2.114 \\
18 & 0.15 & 0.5 & 0.4 & 1.114 & 1.149 & 2.493 & 0.001 & 1.903 \\
20 & 0.15 & 0.5 & 0.4 & 1.121 & 1.092 & 2.420 & 0.001 & 1.687 \\
22 & 0.15 & 0.5 & 0.4 & 1.053 & 1.070 & 2.347 & 0.000 & 1.673 \\
25 & 0.15 & 0.5 & 0.4 & 1.245 & 1.183 & 2.237 & 0.004 & 0.983 \\
30 & 0.15 & 0.5 & 0.4 & 1.081 & 1.087 & 2.053 & 0.000 & 0.945 \\
40 & 0.15 & 0.5 & 0.4 & 1.042 & 1.049 & 1.686 & 0.000 & 0.415 \\
50 & 0.15 & 0.5 & 0.4 & 0.972 & 0.967 & 1.319 & 0.000 & 0.120
\end{tabular}




\section{References}

[1] Abouelatta, O.B. , Madl J., 2001, Surface roughness prediction based on cutting parameters and tool vibrations in turning operations, Journal of Materials Processing Technology, 118, pp. 269277

[2] Akasawa,T., Sakurai, H., Nakamura, M., Tanaka, T., Takano, K. , 2003, Effects of free-cutting additives on the machinability of austenitic stainless steels. Journal of Materials Processing technology, 143 - 144, 66-71

[3] Al-Ahmari A.M.A., 2007, Predictive machinability models for a selected hard material in turning operations. Journal of Material Processing Technology, $190,305-311$

[4] Anthony, X. and Adithan, M. , 2009, Determining the influence of cutting fluids on tool wear and surface roughness during turning of AISI 304 austenitic stainless steel, Journal of Material Processing Technology, 209, 900-909

[5] Baptista, R., Antune Simoes, J.F., 2000, Three and five axis milling of sculptured surfaces, Journal of Materials Processing Technology, 103, pp. 398403

[6] Baykasoglu, A., Dereli, T. , 2002, Novel algorithm approach to generate the number of passes and depth of cuts for the optimization routines of multi pass machining. Int J Prod Res 40: 15491565

[7] Benardos, P.G., Vosniakos, G.-C., 2003, Predicting surface roughness in machining: a review, International Journal of Machine Tools and Manufacture, $43,8,833-844$

[8] Cebeli, O., 2006. Turning of AIS304 austenitic stainless steel, Journal of Engineering and Natural Sciences, 2, 117-121 
[9] Chen MC, Tasi D-M (1996) A simulated annealing approach foroptimiza- tion of multi-pass turning operation. Int J Prod Res 34:28032825

[10] Chryssolouris, G., Guillot, M. ,1990. A comparison of statistical and AI approaches to the selection of process parameters in intelligent machining. ASME J Eng Ind 112:112131

[11] Deb, K., 1995, Optimization for engineering design: algorithms and examples. Prentice-Hall, New York

[12] Glantz SA, Slinker BK. 2000. Primer of Applied Regression and Analysis of Variance. 2nd ed. New York, NY: McGraw-Hill

[13] Gokkaya, Hasan, Nalbant, Muammer. 2007. The effects of cutting tool geometry and processing parameters on the surface roughness of AISI 1030 steel, Materials \& Design, 28 (2)p. 717 - 721

[14] Halevi, G., 2003. Process and Operations Planning. Kluwer Academic Publishers. p.158

[15] Ibrahim, C., 2006. Machining of austenitic stainless steels using CVD multi-layer coated cemented carbide tools, Tribology International, 39(6), 565-569

[16] Jiao, Y., Pei ZS, Lei S, Lee ES, Fisher GR (2005) Fuzzy adaptive networks in machining process modeling dimensional error prediction for turning operations. Int J Prod Res 43:29312948

[17] Kilickap, E., Cakir, O. Aksoy, M. Inan, A., 2005. Study of tool wear and surface roughness in machining of homogenised SiC-p reinforced aluminium metal matrix composite. Journal of Materials Processing Technology. $164165(0)$ p.862-867

[18] Kvanli, Alan H.; Pavur, Robert J.; Keeling, Kellie B. (2006). Concise Managerial Statistics. Engage Learning. pp. 8182

[19] Lehmann, E. L., Casella, George ,1998, Theory of Point Estimation (2nd ed.). New York: Springer 
[20] Munoz-Escalona, P. and Maropoulos, P. G., 2010. Artificial neural networks for surface roughness prediction when face milling Al 7075-T7351. Journal of Materials Engineering and Performance, 19 (2), pp. 185-193.

[21] M'Saoubi R. et al, 2008, A review of surface integrity in machining and its impact on functional performance and life of machined products, International Journal of Sustainable Manufacturing, 1(1/2), 203-236

[22] Ozel T., Hsu T.K., Zeren E., 2005. Effects of cutting edge geometry, workpiece hardness, feed rate and cutting speed on surface roughness and forces in finish turning of hardened AISI H13 steel. Int J Adv Manuf Technol .

25: 262269

[23] Ulas Caydas, Sami Ekici, 2010, Support vector machine models for surface roughness prediction in CNC turning of AISI 304 austenitic stainless steel, Journal of Intelligent Manufacturing 